\title{
Real time cascade adaptive notch filter scheme for sinusoidal parameter estimation
}

\author{
Soo-Chang Pei*, Chien-Cheng Tseng \\ Department of Electrical Engineering, National Taiwan University, Taipei, Taiwan, ROC
}

Received 7 March 1991; revised 25 May 1992 and 2 March 1994

\begin{abstract}
In this paper, a real-time cascade adaptive notch filter scheme for sinusoidal parameter estimation is proposed. The second order recursive maximum likelihood algorithm is used to estimate the sinusoidal frequencies. It is suitable for real-time operations because only 13 multiplications, 14 additions and 1 division are required in each iteration. After adaptive notch filters have converged, the simplified recursive least square algorithm is then used to compute the amplitudes and phases of sinusoids quickly. The error surface analysis indicates that our scheme is unimodal and results in guaranteed convergence. Extensive computer simulations are also included to demonstrate its performance.
\end{abstract}

\section{Zusammenfassung}

In dieser Arbeit wird ein Echtzeitsystem einer adaptiven Kaskade von Kerbfiltern zur Schätzung von Sinus-Parametern vorgeschlagen. Der Maximum Likelihood Algorithmus zweiter Ordnung wird zur Schätzung von Sinusfrequenzen benutzt. Er ist zur Echtzeit-Operation geeignet, da nur 13 Multiplikationen, 14 Additionen und 1 Division in jeder Iteration erforderlich sind. Nachdem die adaptiven Kerbfilter eingelaufen sind, wird der vereinfachte Recursive-leastsquares-Algorithmus zur schnelleren Berechnung der Amplituden und Phasen von Sinussignalen benutzt. Die Fehleranalyse zeigt, daß unser System unimodal ist und Konvergenz garantiert. Ausführliche Computer Simulationen werden zur Demonstration der Eigenschaften angeführt.

\section{Résumé}

Dans cet article, un schéma temps-réel basé sur des filtres 'notchs' adaptatifs en cascade pour l'estimation de paramètres sinusoidaux est proposé. L'algorithme de maximum de vraisemblance récursif du second ordre est utilisé pour l'estimation des fréquences sinusoidales. Il est adapté pour les opérations temps-réels car seulement 13 multiplications, 14 additions et 1 division sont requise à chaque itération. Une fois que les filtres adaptatifs 'notch' ont convergés, l'algorithme récursif simplifié d'estimation aux moindres carrés est alors utilisé pour calculer rapidement les amplitudes et les phases des sinusoides. L'analyse de la surface d'erreur indique que notre schéma est unimodal et que la convergence est guarantie. De nombreuses simulations sur ordinateurs sont également incluses pour démontrer les performances du schéma.

Key words: Adaptive notch filter; Sinusoidal parameter estimation

\footnotetext{
*Corresponding author.
} 


\section{Introduction}

Adaptive notch filters are very useful in many signal processing applications such as the retrieval of sinusoids in noise, eliminating sinusoidal power line disturbance in a measurement signal. Examples are in the areas of radar, communications, control, biomedical engineering and others. In early work, most adaptive notch filters are implemented as direct form high order IIR filters [1, 8-10]. This form suffers from two drawbacks. One is that stability monitoring is difficult, the other is, the frequencies of the sinusoids need to be determined from the filter coefficients by using root finding. Thus, the cascade form adaptive notch filters are developed in the literature [3-6].

Recently Martin and M'sirdi both have used cascade adaptive notch filter schemes to estimate sinusoidal parameters in real time independently. In this paper, we propose another scheme to solve the same problem. There are three main differences between our scheme and theirs. First, Martin and M'sirdi used the original signal as reference to update amplitude and phase, but we used the enhanced signal as reference. As a result, our scheme is more accurate in amplitude and phase estimation than theirs. Second, M'sirdi used conventional recursive least square (RLS) algorithm to estimate amplitude and phase, but we will show that the RLS algorithm can be simplified. Third, error surface analysis indicates that Martin's scheme is multimodal, but ours is unimodal. Thus, Martin's scheme may converge to local minima.

This paper is organized as follows. First, the real-time cascade adaptive notch filter scheme is developed in Section 2. Next, error surface analysis is presented in Section 3. Finally, some computer simulations are demonstrated in Section 4.

\section{Cascade adaptive notch filter scheme}

Consider the following noisy sinusoidal signals.

$$
y(t)=\sum_{i=1}^{p} A_{i} \cos \left(\omega_{i} t+\phi_{i}\right)+v(t)=\sum_{i=1}^{p} s_{i}(t)+v(t)
$$

where $\omega_{i}$ is the unknown angular frequency, $A_{i}$ is sinusoidal amplitude, $\phi_{i}$ is an initial phase, and $v(t)$ is zero-mean white noise with variance $\sigma^{2}$. Assume that the number of the sinusoids $p$ is known in advance. The problem considered in this paper is as follows. Given the noisy samples $y(t)$, using the cascade adaptive notch filter scheme, estimate $\omega_{i}$, $A_{i}$, and $\phi_{i}$ in real time.

\subsection{Adaptive notch filter scheme}

The proposed adaptive notch filter scheme is shown in Fig. 1 for $p=2$ case. The major building blocks of the scheme are several second-order adaptive notch filters and amplitude/phase estimators. The estimation procedures of this scheme can be divided into the following two steps. First, use cascade adaptive notch filters with recursive maximum likelihood (RML) algorithm to extract every sinusoidal signal $\hat{s}_{i}(t)$ from input signal $y(t)$ and to estimate sinusoidal frequencies $\hat{\omega}_{i}$. Second, each estimated frequencies $\hat{\omega}_{i}$ and enhanced sine wave $\hat{s}_{i}(t)$ are sent to the amplitude/phase estimator in order to obtain estimate values of amplitude and phase. Now, let us describe every building block in the following.

\subsection{Second-order adaptive notch filter with RML algorithm}

The ith second order notch filter in Fig. 1 is realized by

$H_{i}(z)=\frac{1+a_{i} z^{-1}+z^{-2}}{1+r a_{i} z^{-1}+r^{2} z^{-2}}$

which is also used by Nehorai [8] and M'sirdi $[5,6]$. Its notch frequency $\omega_{\mathrm{N}}^{i}$ and $3 \mathrm{~dB}$ rejection bandwidth $B W$ are given by

$\omega_{\mathrm{N}}^{i}=\arccos \left(\frac{-a_{i}}{2}\right)$,

$B W=\pi(1-r)$.

Let the input and output signals of notch filter $H_{i}(z)$ be $y_{i}(t)$ and $e_{i}(t)$ respectively. Then notch filter 


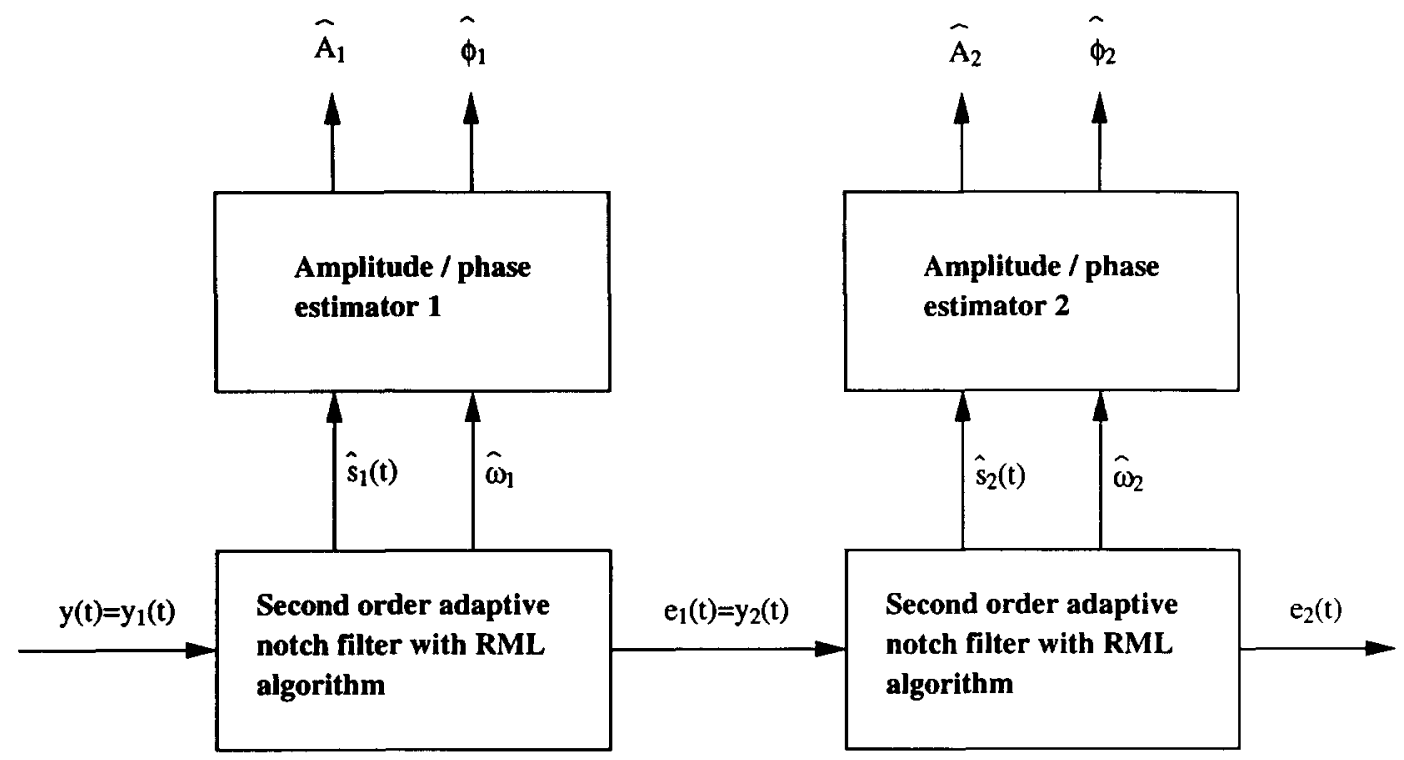

Fig. 1. Proposed adaptive notch filter scheme for sinusoidal parameter estimation.

parameter $a_{i}$ is updated by minimizing the cost function

$J\left(a_{i}, t\right)=\sum_{n=1}^{t} \lambda^{t-n} e_{i}(n)$

Because only second-order notch filter is used here, conventional high order RML algorithm can be simplified as follows [8].

The second-order adaptive notch filter with $R M L$ algorithm

\section{Input signal: $\quad y_{i}(t)$}

Output signal: $\quad \hat{s}_{i}(t), e_{i}(t)$

Initialization: $\quad a_{i}(0)=0, p_{i}(0)=\frac{100}{E\left(y_{i}^{2}(t)\right)}$

$$
\begin{aligned}
& \psi_{i}(-1)=\psi_{i}(0)=0, \\
& y_{i}(-1)=y_{i}(0)=0 \\
& e_{i}(-1)=e_{i}(0)=0, \\
& \lambda(1)=0.9, \lambda_{0}=0.99, \\
& r_{0}=0.99, r(1)=0.8, r_{\infty}=0.99
\end{aligned}
$$

Main loop:

(1) $\phi_{i}(t)=r(t) e_{i}(t-1)-y_{i}(t-1)$

(2) $\psi_{i}(t)=\phi_{i}(t)-a_{i}(t-1) r(t) \psi_{i}(t-1)$

$$
-r^{2}(t) \psi_{i}(t-2)
$$

(3) $\bar{e}_{i}(t)=y_{i}(t)+y_{i}(t-2)$

$$
-r^{2}(t) e_{i}(t-2)-a_{i}(t-1) \phi_{i}(t)
$$

(4) $p_{i}(t)=\frac{p_{i}(t-1)}{\lambda(t)+p_{i}(t-1) \psi_{i}^{2}(t)}$

(5) $a_{i}(t)=a_{i}(t-1)+p_{i}(t) \psi_{i}(t) \bar{e}_{i}(t)$

(6) $e_{i}(t)=y_{i}(t)+y_{i}(t-2)$

$$
-r^{2}(t) e_{i}(t-2)-a_{i}(t) \phi_{i}(t)
$$

(7) $\hat{s}_{i}(t)=y_{i}(t)-e_{i}(t)$

(8) $\lambda(t+1)=\lambda_{0} \lambda(t)+\left(1-\lambda_{0}\right)$

(9) $r(t+1)=r_{0} r(t)+\left(1-r_{0}\right) r_{\infty}$

End loop.

Note that the frequency estimate $\hat{\omega}_{i}$ can be evaluated from Eq. (3). This algorithm takes only 13 multiplications, 14 additions, and 1 division in each iteration, so it is suitable for real time operations. Also, the RML algorithm is an asymptotically efficient parameter estimation algorithm, so its accuracy is high.

\subsection{Amplitude/phase estimator}

Assume that the estimated frequency and enhanced sine wave received from adaptive notch 
filter are $\hat{\omega}_{i}$ and $\hat{s}_{i}(t)$, respectively. Using both signals, the following vector equation may be written.

$X_{t} \theta_{t}=C_{t}$,

where

$X_{t}=\left[\begin{array}{llll}\cos \left(1 \hat{\omega}_{i}\right) & \cos \left(2 \hat{\omega}_{i}\right) & \cdots & \cos \left(t \hat{\omega}_{i}\right) \\ \sin \left(1 \hat{\omega}_{i}\right) & \sin \left(2 \hat{\omega}_{i}\right) & \cdots & \sin \left(t \hat{\omega}_{i}\right)\end{array}\right]^{\mathrm{T}}$,

$\theta_{t}=\left[\begin{array}{ll}a(t) & b(t)\end{array}\right]^{\mathrm{T}}$,

$C_{t}=\left[\begin{array}{llll}\hat{s}_{i}(1) & \hat{s}_{i}(2) & \cdots & \hat{s}_{i}(t)\end{array}\right]^{\mathrm{T}}$.

Notice that M'sirdi and Martin use input signal $y(t)$ to construct vector $C_{t}[4,6]$, i.e.,

$C_{t}=\left[\begin{array}{llll}y_{i}(1) & y_{i}(2) & \cdots & y_{i}(t)\end{array}\right]^{\mathrm{T}}$.

This is a difference between our scheme and theirs.

Then the well-known least square solution is given by

$$
\begin{aligned}
\theta_{t} & =\left(X_{t}^{\mathrm{T}} X_{t}\right)^{-1} X_{t}^{\mathrm{T}} C_{t} \\
& =R(t)^{-1} D(t),
\end{aligned}
$$

where

$R(t)=\frac{2}{t} X_{t}^{\mathrm{T}} X_{t}$

$D(t)=\frac{2}{t} X_{t}^{\mathrm{T}} C_{t}$.

Thus, the desired amplitude and phase estimates are given by

$\hat{A}_{i}=\sqrt{a^{2}(t)+b^{2}(t)}$,

$\hat{\phi}_{i}=\arctan \left(\frac{-b(t)}{a(t)}\right)$.

Because cosine and sine are orthogonal, it is easy to show that

$\lim _{t \rightarrow \infty} R(t)=I$,

where $I$ is the identity matrix. This expression means that when $t$ increases $R(t)$ converges to identity matrix. Thus, computing $R(t)$ is redundant when $t$ is large. It can be omitted. Therefore, the least square solution can be reduced to

$\theta_{t}=D(t)$
Moreover, $D(t)$ obeys the following recursive formula:

$D(t)=\frac{t-1}{t} D(t-1)+\frac{2}{t} \hat{s}_{i}(t)\left[\begin{array}{cc}\cos \left(\hat{\omega}_{i} t\right) & \sin \left(\hat{\omega}_{i} t\right)\end{array}\right]^{\mathrm{T}}$.

Finally, we propose a simplified RLS algorithm to estimate amplitude and phase as follows:

\section{Simplified RLS Algorithm}

Input: $\quad \hat{\omega}_{i}$ and $\hat{s}_{i}(t)$

Output: $\quad \hat{A}_{i}(t)$ and $\hat{\phi}_{i}(t)$

Initialization: $\theta_{i}(0)=\left[\begin{array}{ll}0 & 0\end{array}\right]^{\mathbf{T}}$

Main Loop:

$$
\begin{aligned}
& \text { (1) } \begin{aligned}
\theta(t) & =\alpha_{i} \theta(t-1)+2\left(1-\alpha_{t}\right) \\
& \times \hat{s}_{i}(t)\left[\cos \left(\hat{\omega}_{i} t\right) \sin \left(\hat{\omega}_{i} t\right)\right]^{\mathrm{T}}
\end{aligned} \\
& \text { (2) } \begin{aligned}
A_{i}(t) & =\sqrt{a^{2}(t)+b^{2}(t)}
\end{aligned} \text { (3) } \phi_{i}(t)=\arctan \left(\frac{-b(t)}{a(t)}\right)
\end{aligned}
$$

End Loop.

Note that $\alpha_{t}=(t-1) / t$. However, in order to produce a forgetting effect, $\alpha_{t}$ may be fixed to be a constant. In the following simulation we choose $\alpha_{t}=0.99$.

\subsection{A Comparison to other schemes}

(A) Accuracy: In amplitude and phase estimation, we use enhanced signal $\hat{s}_{i}(t)$ as reference signal, but M'sirdi and Martin use original signal $y(t)$ as reference. Also, signal to noise ratio (SNR) improvement factor [8] is

$\frac{\operatorname{SNR}\left(\hat{s}_{i}(t)\right)}{\operatorname{SNR}(y(t))}=\frac{1}{1-r}$,

so SNR of $\hat{s}_{i}(t)$ is much higher than SNR of $y(t)$ when $r$ approaches unity. As a result, our scheme has better accuracy than M'sirdi's scheme in amplitude and phase estimation.

(B) Complexity: Because M'sirdi uses conventional RLS algorithm to update amplitude and phase, its complexity is proportional to $p^{2}$. But we 
use simplified RLS algorithm to estimate amplitude and phase, so complexity is only linear with $p$. Thus, M'sirdi's scheme is less efficient than ours in complexity.

(C) A simple example: In order to compare our scheme with M'sirdi's scheme more clearly, a Monte Carlo simulation is tested under input data length 1000 and different SNR on the VAX computer. The input signal is

$y(t)=A \cos \left(0.2 \pi t+\frac{\pi}{2}\right)+A \cos \left(0.3 \pi t+\frac{\pi}{2}\right)+v(t)$

where $A$ is determined in term of SNR. Table 1 and Table 2 summarize the bias and standard deviation of sinusoidal parameters calculated from 40 independent trials. From these results it is easy to see that our scheme has better statistical accuracy than M'sirdi's scheme. Moreover, the CPU time of each trial in our scheme is $1.18 \mathrm{~s}$, but the CPU time in M'sirdi's scheme is $1.48 \mathrm{~s}$. Thus, our scheme is superior to M'sirdi's scheme in both accuracy and complexity.

\section{Error surface analysis of cascade adaptive notch filters}

Error surface analysis is very useful for understanding the convergence of adaptive recursive filters and provides valuable insight $[7,11]$. There are

Table 1

Statistical results of M'sirdi's scheme

\begin{tabular}{|c|c|c|c|c|c|c|c|}
\hline $\begin{array}{l}\text { SNR } \\
\text { (dB) }\end{array}$ & $N$ & $\hat{A}_{1}$ & $\hat{f}_{1}$ & $\hat{\phi}_{1}$ & $\hat{A_{2}}$ & $\hat{f}_{2}$ & $\tilde{\phi}_{2}$ \\
\hline \multicolumn{8}{|c|}{ Bias $\times 10^{-4}$} \\
\hline 3 & & 157.1 & 0.0846 & -230.8 & 689.8 & 0.305 & 245.5 \\
\hline 6 & & 142.4 & 0.0806 & -205.8 & 455.7 & 0.258 & 174.5 \\
\hline 9 & 1000 & 114.8 & 0.0605 & -194.8 & 385.8 & 0.209 & -144.3 \\
\hline 12 & & 91.28 & 0.0425 & -189.4 & 335.6 & 0.201 & -108.1 \\
\hline \multicolumn{8}{|c|}{ Standard deviation $\times 10^{-4}$} \\
\hline 3 & & 900.8 & 0.5384 & 491.1 & 1091 & 0.5612 & 446.9 \\
\hline 6 & & 897.6 & 0.3752 & 349.7 & 1061 & 0.3992 & 336.9 \\
\hline 9 & 1000 & 887.4 & 0.2672 & 248.8 & 1053 & 0.3024 & 274.8 \\
\hline 12 & & 882.1 & 0.2132 & 185.3 & 1026 & 0.2063 & 193.8 \\
\hline
\end{tabular}

Table 2

Statistical results of our scheme

\begin{tabular}{|c|c|c|c|c|c|c|c|}
\hline $\begin{array}{l}\text { SNR } \\
\text { (dB) }\end{array}$ & $N$ & $\hat{A}_{1}$ & $\hat{f}_{1}$ & $\hat{\phi}_{1}$ & $\hat{A}_{2}$ & $\hat{f}_{2}$ & $\hat{\phi}_{2}$ \\
\hline \multicolumn{8}{|c|}{ Bias $\times 10^{-4}$} \\
\hline 3 & & 115.8 & 0.0846 & -212.1 & -517.2 & 0.305 & -161.7 \\
\hline 6 & & 84.99 & 0.0806 & -195.3 & -352.3 & 0.258 & -133.6 \\
\hline 9 & 1000 & -82.63 & 0.0605 & -179.4 & -296.1 & 0.209 & -109.8 \\
\hline 12 & & 45.76 & 0.0425 & -174.7 & -253.2 & 0.201 & -104.9 \\
\hline \multicolumn{8}{|c|}{ Standard deviation $\times 10^{-4}$} \\
\hline 3 & & 797.8 & 0.5384 & 429.6 & 726.4 & 0.5612 & 395.8 \\
\hline 6 & & 777.2 & 0.3752 & 303.5 & 712.7 & 0.3992 & 299.6 \\
\hline 9 & 1000 & 768.1 & 0.2672 & 213.9 & 706.9 & 0.3024 & 245.5 \\
\hline 12 & & 750.8 & 0.2132 & 164.5 & 679.9 & 0.2063 & 174.5 \\
\hline
\end{tabular}




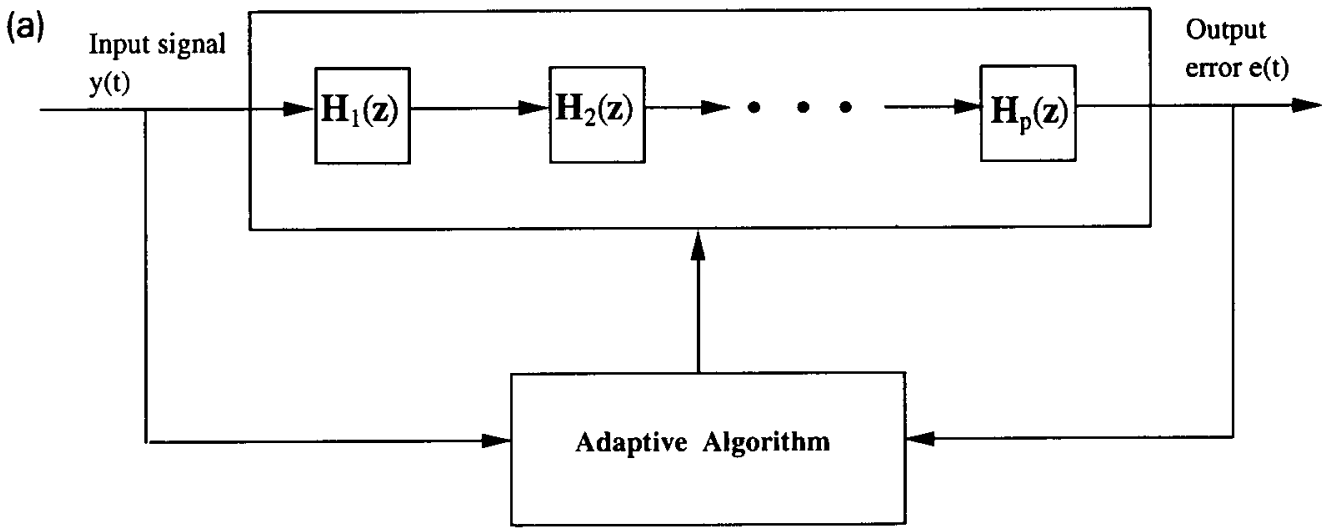

Fig. 2(a). Simultaneous adaption scheme: the cascade second-order adaptive notch filters are updated simultaneously.

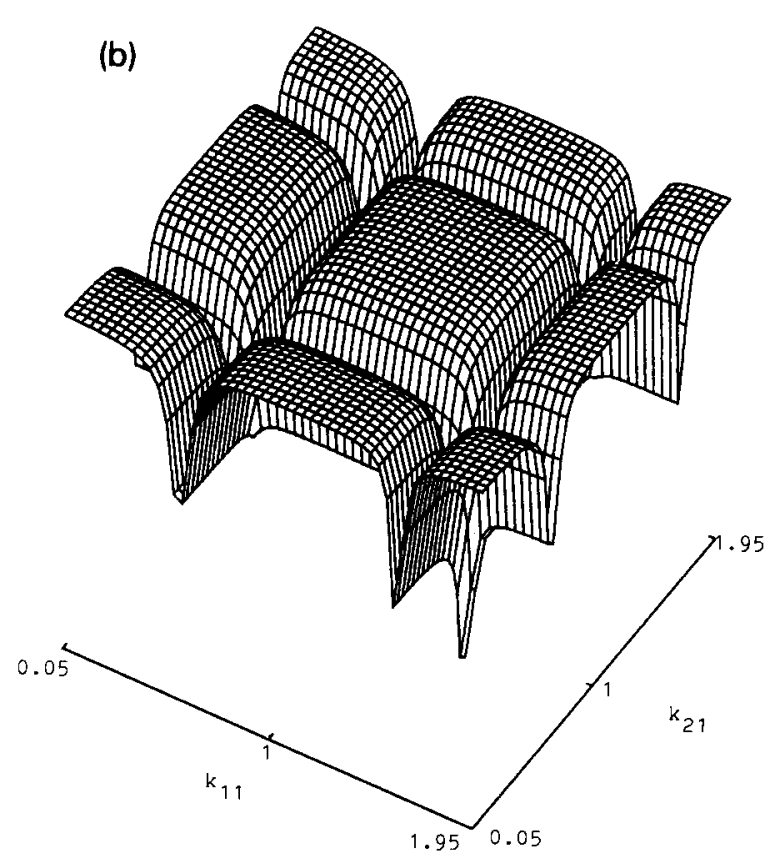

Fig. 2(b). Three-dimensional plot of the mean square error $E\left(e^{2}(t)\right)$ versus the notch filter parameters $k_{11}$ and $k_{21}$ for two sinusoids inputs $(p=2)$.

two different ways to adjust cascade adaptive notch filter's parameters. One is that the filter coefficients are updated simultaneously for all stages such as Martin's scheme. The other is that the filter coefficients are updated individually in each stage, for example in M'sirdi's and our schemes. Until now, comparison between these two adaptions has not been very clear and has not appeared in the open literature. In this section, we will use the error surface analysis to discuss their difference. The results tell us the error surface of simultaneous adaption scheme is multi-dimensional and has local minima. And the error surface of individual adaptation is strictly one-dimensional, unimodal and has guaranteed convergence. The onedimensional solution search space in individual adaption is much smaller and easier to handle than the multi-dimensional one in simultaneous adaption.

\subsection{Simultaneous adaptive scheme}

Fig. 2(a) shows the system that the cascade adaptive notch filters are updated simultaneously. The input signal consists of $p$ sinusoids in addition to white noise, as expressed in Eq. (1). Martin has chosen a low coefficient sensitivity biquad as desired second-order notch filter, i.e.

$$
H_{i}(z)=\frac{2-k_{i 2}}{2} \frac{1-\frac{2\left(2-k_{i 2}-k_{11}^{2}\right)}{2-k_{i 2}} z^{-1}+z^{-2}}{1-\left(2-k_{i 2}-k_{i 1}^{2}\right) z^{-1}+\left(1-k_{i 2}\right) z^{-2}}
$$

where $k_{i 2}=1-r^{2} \quad$ and $\quad k_{i 1}=2 \sqrt{1-\left(k_{i 2} / 2\right)}$ $\sin \left(\omega_{\mathrm{N}}^{i} / 2\right)$ if $r$ is pole radius and $\omega_{\mathrm{N}}^{i}$ is notch 
(c)

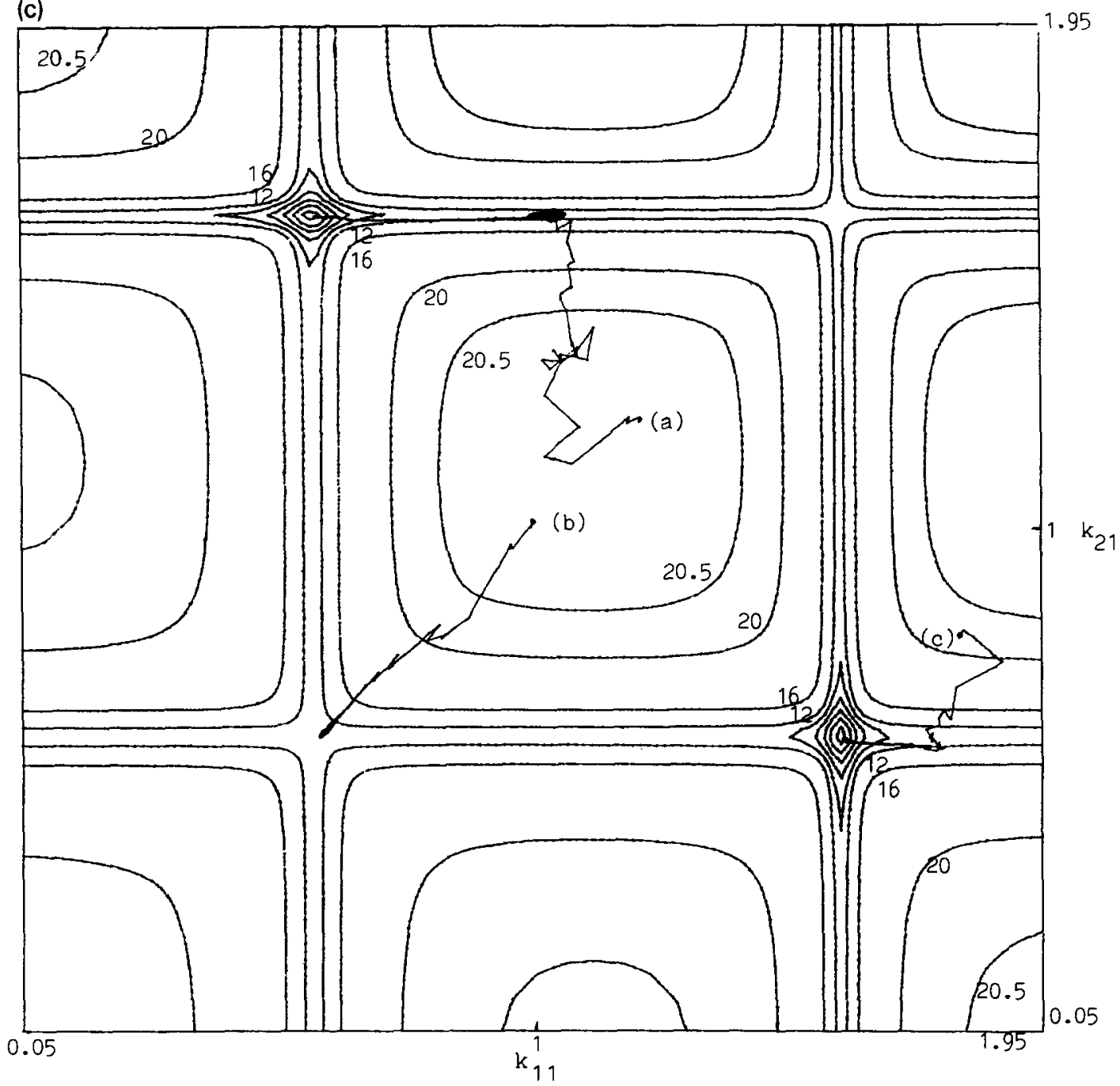

Fig. 2(c). Error surface contour plot and the trajectories of three simulations initialized at the points $\left(k_{11}, k_{21}\right)=(\mathrm{a})(1.2,1.2),(\mathrm{b})(1.0,1.0)$ and $(c)(1.8,0.8)$ respectively.

frequency. The objective of the simultaneous adaptation algorithm is to adjust the $k_{i 1}$ 's such that the output mean square error $E\left(e^{2}(t)\right)$ is minimized. During adaption, $3 \mathrm{~dB}$ rejection bandwidth is kept constant by choosing a fixed value for $k_{i 2}$ 's. It is easy to show that the mean square error is

$$
E\left(e^{2}(t)\right)=\sum_{i=1}^{p} \frac{A_{i}^{2}}{2}\left|H\left(\mathrm{e}^{\mathrm{j} \omega_{i}}\right)\right|^{2}+\frac{\sigma^{2}}{2 \pi \mathrm{j}} \oint_{c}|H(z)|^{2} \frac{\mathrm{d} z}{z},
$$

where $c$ is a counterclockwise unit circle contour, and $H(z)=\prod_{i=1}^{p} H_{i}(z)$. The first term of Eq. (13) is closed-form formula, we can calculate it exactly by computer. However, the second term is difficult to simplify by the Cauchy's residue theorem, so we use numerical integration, such as Trapezoid rule, to compute its approximate value through the following equation:

$\frac{\sigma^{2}}{2 \pi \mathrm{j}} \oint_{c}|H(z)|^{2} \frac{\mathrm{d} z}{z}=\frac{\sigma^{2}}{2 \pi} \int_{-\pi}^{\pi}\left|H\left(\mathrm{e}^{\mathrm{j} \omega}\right)\right|^{2} \mathrm{~d} \omega$. 
Now, we consider an example with the input signal as

$y(t)=\sqrt{20} \cos (0.2 \pi t)+\sqrt{20} \cos \left(0.6 \pi t+\frac{\pi}{2}\right)+v(t)$.

A 3D plot of the mean square error $E\left(e^{2}(t)\right)$ versus the two notch parameters $k_{11}, k_{21}$ is depicted in Fig. 2(b) for $r=0.95$. From the plot, we observe that the local minima are at four ditches $k_{11}=0.603, \quad k_{11}=1.579, \quad k_{21}=0.603, \quad$ and $k_{21}=1.579$. When the notch parameters are updated and converge to one of these four ditches, the cascade adaptive notch filter can only eliminate one sinusoid, while the other will still exist. Furthermore, we observe that there are two minima at deep troughs $\left(k_{11}, k_{21}\right)=(0.603,1.579)$ and $(1.579$, 0.603 ). When the notch parameters are adjusted and converge to one of two troughs, the cascade adaptive notch filter can eliminate two sinusoids successfully. In order to understand the convergence behavior of Martin's algorithm, we use his algorithm to adjust the parameters of notch filter. These examples are illustrated in Fig. 2(c). Initial points of the three examples are $\left(k_{11}, k_{12}\right)=$ $(1.2,1.2),(1.0,1.0)$, and $(1.8,0.8)$. In each example, the parameters are adjusted by 1000 iterations. As a result, we find that the trajectory initialized at $(1.0,1.0)$ converges to $(0.603,0.603)$. Even though it is updated 4000 iterations, it still stays there. This situation means that the two notch stages both converge to the same sinusoid's frequency which is not the desired minimum solution. From the above description, we know that there are two local minimums at $(0.603,0.603)$ and $(1.579,1.579)$. Therefore, mean square error surface of cascade adaptive notch filter which is updated simultaneously is multimodal, so a judicious choice of the initial parameters is important.

\subsection{Individual adaptive scheme}

Fig. 3(a) shows the system that cascade adaptive notch filters are updated individually. The input signal is still $p$ sinusoids in an additive white noise as above. The second-order notch filter used in
M'sirdi's scheme and ours is $H_{i}(z)$ which is expressed in Eq. (2). In each stage, the objective of the adaptive algorithm is to adjust $a_{i}$ such that the mean square error $E\left(e_{i}^{2}(t)\right)$ at each stage is minimized. For the first stage, it is easy to show that

$$
E\left(e_{1}^{2}(t)\right)=\sum_{i=1}^{p} \frac{A_{i}^{2}}{2}\left|H_{1}\left(\mathrm{e}^{\mathrm{j} \omega_{i}}\right)\right|^{2}+\frac{\sigma^{2}}{2 \pi \mathrm{j}} \oint_{c}\left|H_{1}(z)\right|^{2} \frac{\mathrm{d} z}{z}
$$

Fig. 3(a) shows the error surface of $E\left(e_{1}^{2}(t)\right)$ versus $a_{1}$ when the input signal is

$$
y(t)=\sqrt{20} \cos (0.2 \pi t)+\sqrt{20} \cos \left(0.6 \pi t+\frac{\pi}{2}\right)+v(t) .
$$

It is interesting to see that the error surface is one-dimensional and has two distinct minima associated with each frequencies, so the first stage will converge to one of the two sinusoid's frequencies. Because the second order notch filter's frequency response is unity gain and zero phase everywhere except near the notch frequency, the other sinusoid signal and noise pass through the filter $H_{1}(z)$ almost undistortedly. Thus the mean square output error of the second stage is

$E\left(e_{2}^{2}(t)\right)=\sum_{i=1, i \neq k}^{p} \frac{A_{i}^{2}}{2}\left|H_{2}\left(\mathrm{e}^{\mathrm{j} \omega_{i}}\right)\right|^{2}+\frac{\sigma^{2}}{2 \pi j} \oint_{c}\left|H_{2}(z)\right|^{2} \frac{\mathrm{d} z}{z}$

where $k$ is the sinusoid removed by the first stage. The whole situation of the second stage is the same as the first stage, and one of the other sinusoids will be eliminated by the second stage. Repeat this process until all of the sinusoids are eliminated individually in each stage. Fig. 3(c) shows the learning curves of the parameters $a_{i}, i=1,2$ in the two stages when the input $y(t)$ is expressed in Eq. (17). This figure reveals that two sinusoidal frequencies can be estimated correctly and quickly by cascading notch filters. Therefore, the error surface of second order notch filter which is updated individually is unimodal and has no local minima. 
(a)
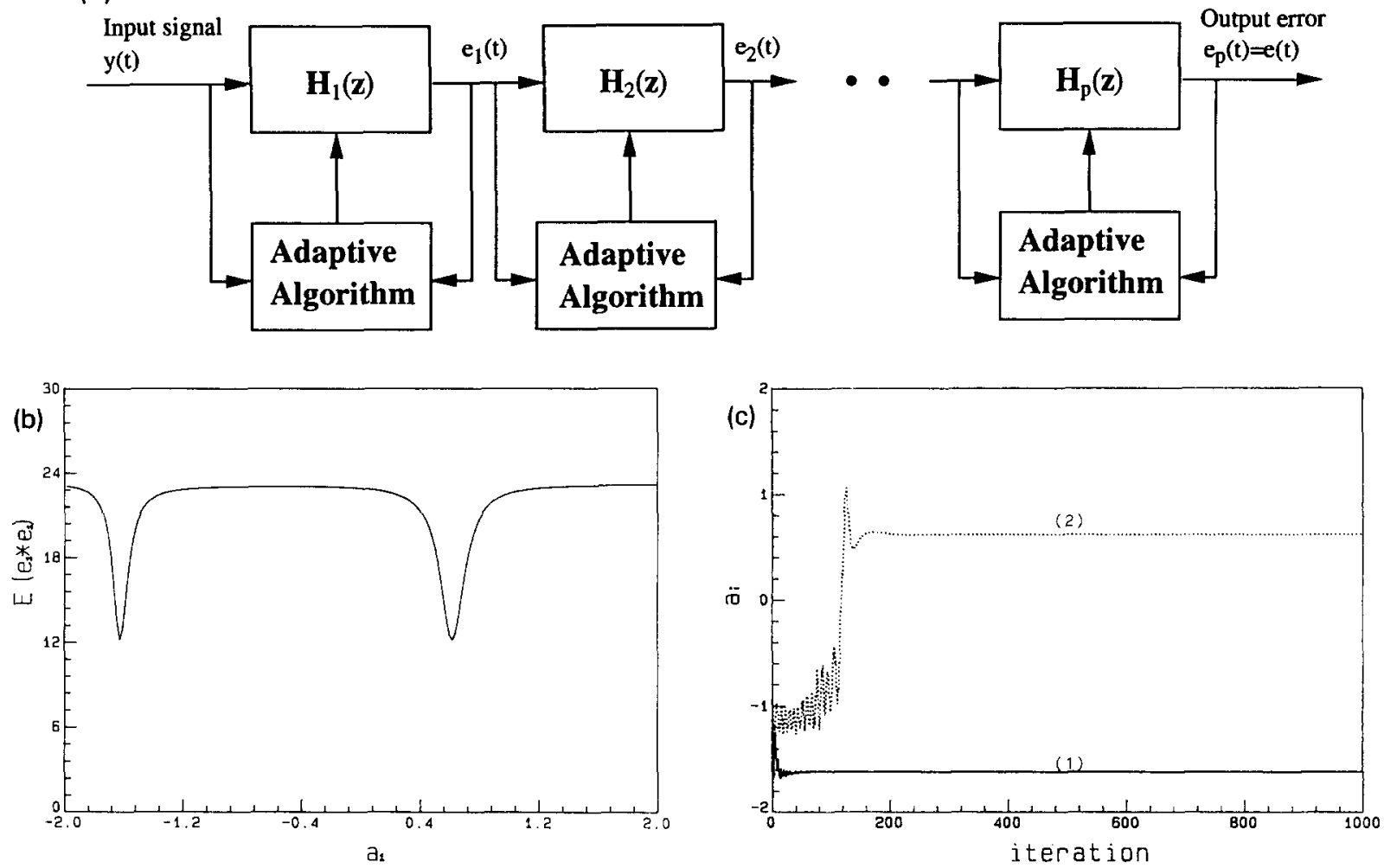

Fig. 3. (a) Individual adaption scheme: the cascade second order adaptive notch filters are updated individually. (b) The error surface plot of the mean square error $E\left(e_{1}^{2}(t)\right)$ versus the notch filter parameter $a_{1}$ for two sinusoidal inputs. (c) The learning curves of the two stage notch filter parameters $a_{1}$ and $a_{2}$ for two input sinusoids. [(1) solid line: first stage, (2) dot line: second stage]

\section{Computer simulations}

Some experiments are run on the VAX $11 / 780$ computer to test the proposed scheme for sinusoidal parameter estimation and the tracking performance with RML algorithm.

Example 1. (Tracking stationary input). In the example, the input signal is

$y(t)=3 \cos \left(0.1 \pi t+\frac{\pi}{3}\right)+2 \cos \left(0.2 \pi t+\frac{\pi}{2}\right)+v(t)$

Fig. 4 shows the learning curves of each sinusoidal parameters of proposed scheme respectively. As a result, both stages converge to the correct solution as expected.

Example 2. (Tracking non-stationary input). In the example, we will study the tracking behavior of proposed scheme for the non-stationary input. We make some comparisons with Martin's simultaneous adaption scheme in a various condition. The scenario of the experiment is similar to the Examples $1-4$ in Ref. [3]. Four different cases are invistigated as follows:

(A) Two sinusoids are not close to each other in frequency. Let the input signal be

$y(t)=$

$\begin{cases}\sqrt{20} \cos (0.3 \pi t)+\sqrt{20} \cos (0.2 \pi t)+v(t) & \text { if } t \leqslant 400, \\ \sqrt{20} \cos (0.4 \pi t)+\sqrt{20} \cos (0.2 \pi t)+v(t) & \text { if } t>400 .\end{cases}$ 

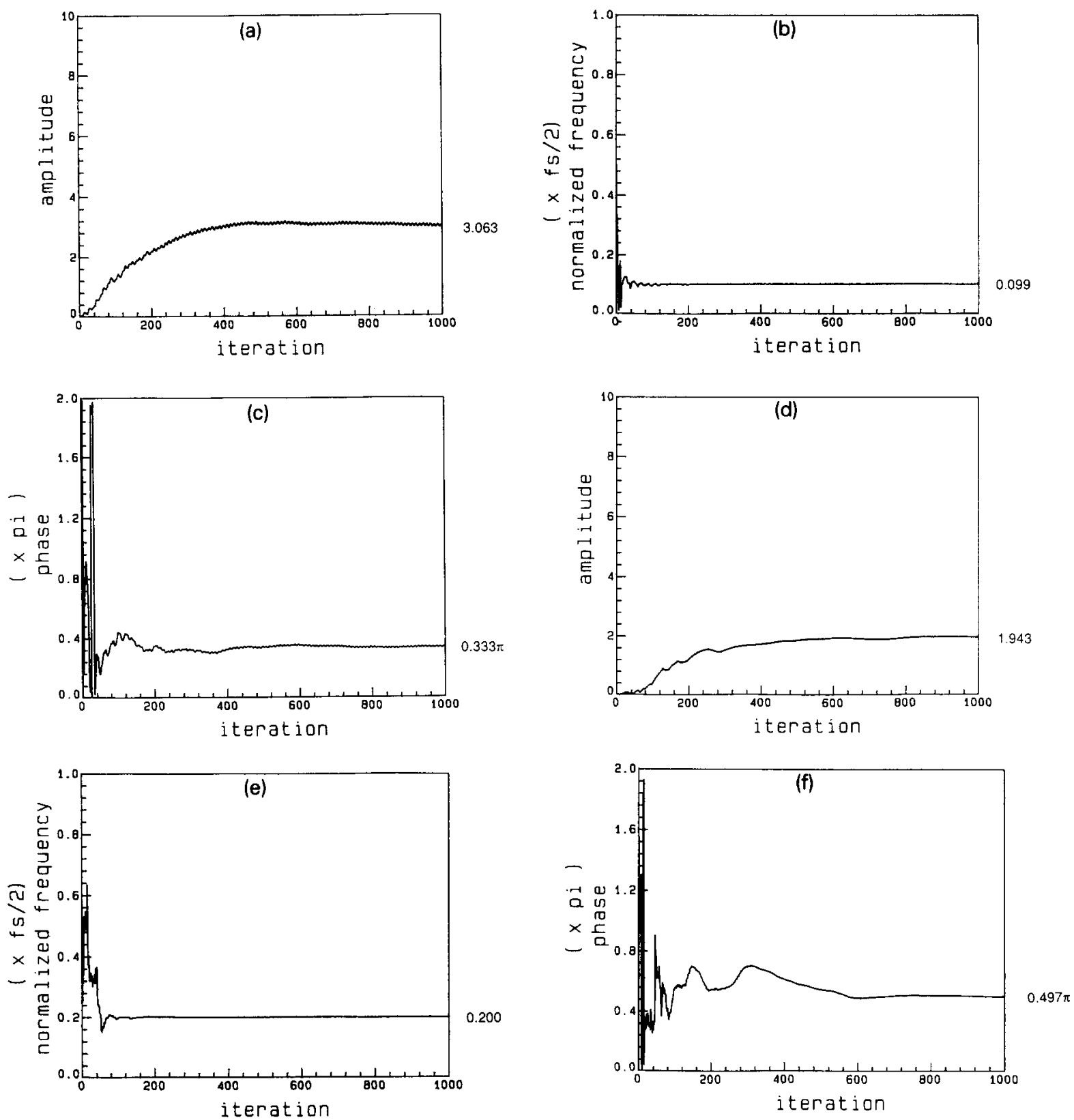

Fig. 4. (a) The learning curve of the amplitude in the first stage. (b) The learning curve of the frequency in the first stage. (c) The learning curve of the phase in the first stage. (d) The learning curve of the amplitude in the second stage. (e) The learning curve of the frequency in the second stage. (f) The learning curve of the phase in the second stage.

After 400 samples, one sinusoid's normalized frequency is changed from 0.3 to 0.4 suddenly. Fig. 5 shows the learning curve of frequencies of 2 stage second-order system. The first stage converges to the sinusoid with frequency 0.3 in the beginning and then tracks the sinusoid at 0.4 quickly. The second stage always deletes the sinusoid at 0.2 without change. 


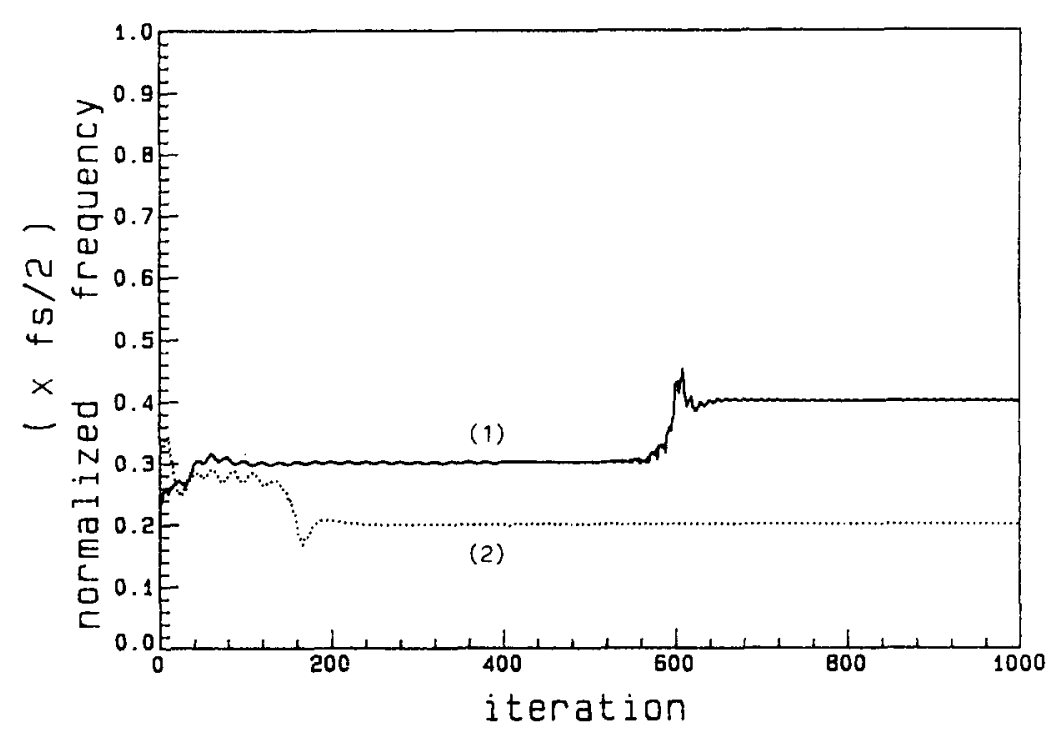

Fig. 5. The learning curves of frequencies of 2 stage second-order systems for two input step changed sinusoids which are separated in frequencies. [(1) solid line: first stage, (2) dot line: second stage]

(B) (Two sinusoids are close to each other in frequency.) The input signal is

$y(t)=$

$\begin{cases}\sqrt{20} \cos (0.3 \pi t)+\sqrt{20} \cos (0.25 \pi t)+v(t) & \text { if } t \leqslant 400 \\ \sqrt{20} \cos (0.4 \pi t)+\sqrt{20} \cos (0.25 \pi t)+v(t) & \text { if } t>400 .\end{cases}$

Two sinusoids in this example are closely spaced at normalized frequencies of 0.25 and 0.3 . Refering to Fig. 6(a), the first stage converges to 0.3 and the second stage converges to 0.25 in the beginning. Also, a 'ripple' effect occurs in the first stage because of the influence of nearby sinusoid at 0.25 . When the input frequency at 0.3 is step changed to 0.4 after 400 samples, the first stage starts tracking and switches converged frequency from 0.3 to 0.25 . This is because the frequency at 0.3 is much closer to 0.25 than the frequency at 0.4 . It will force the second stage to change converged frequency from 0.25 to 0.4 . This means that the first stage has higher priority than the second one during the adaption process. In addition, the same situation is tested for Martin's simultaneous adaption scheme. Refering to Fig. 6(b), the result is interesting and quite different from Fig. 6(a). The first stage changes converged frequency from 0.3 to the dis- tant frequency at 0.4 instead of the close one at 0.25 , and the second stage always converge at 0.25 without change. This is because the simultaneous adaption algorithm minimizes the final output mean square error. The advantages of Martin's scheme are that it has no 'ripple' effect and it has global tracking mechanism. However, large complexity and heavy computation are required for Martin's scheme. Simplicity and comparable performance can be obtained by our individual adaption scheme.

(C) (Two sinusoids are close in frequency with large power difference.) The input signal is

$$
y(t)=\sqrt{200} \cos (0.24 \pi t)+\sqrt{2} \cos (0.28 \pi t)+v(t) .
$$

The input consists of two closely spaced sinusoids with SNR of 20 and $0 \mathrm{~dB}$ at frequencies 0.24 and 0.28 respectively. Refering to Fig. 7 , after the first stage converges to the large power sinusoid at 0.24 , the second stage quickly tracks the sinusoid with the smaller power. No ripple effect is seen in this case due to the very little effect with the smaller power sinusoid has. 

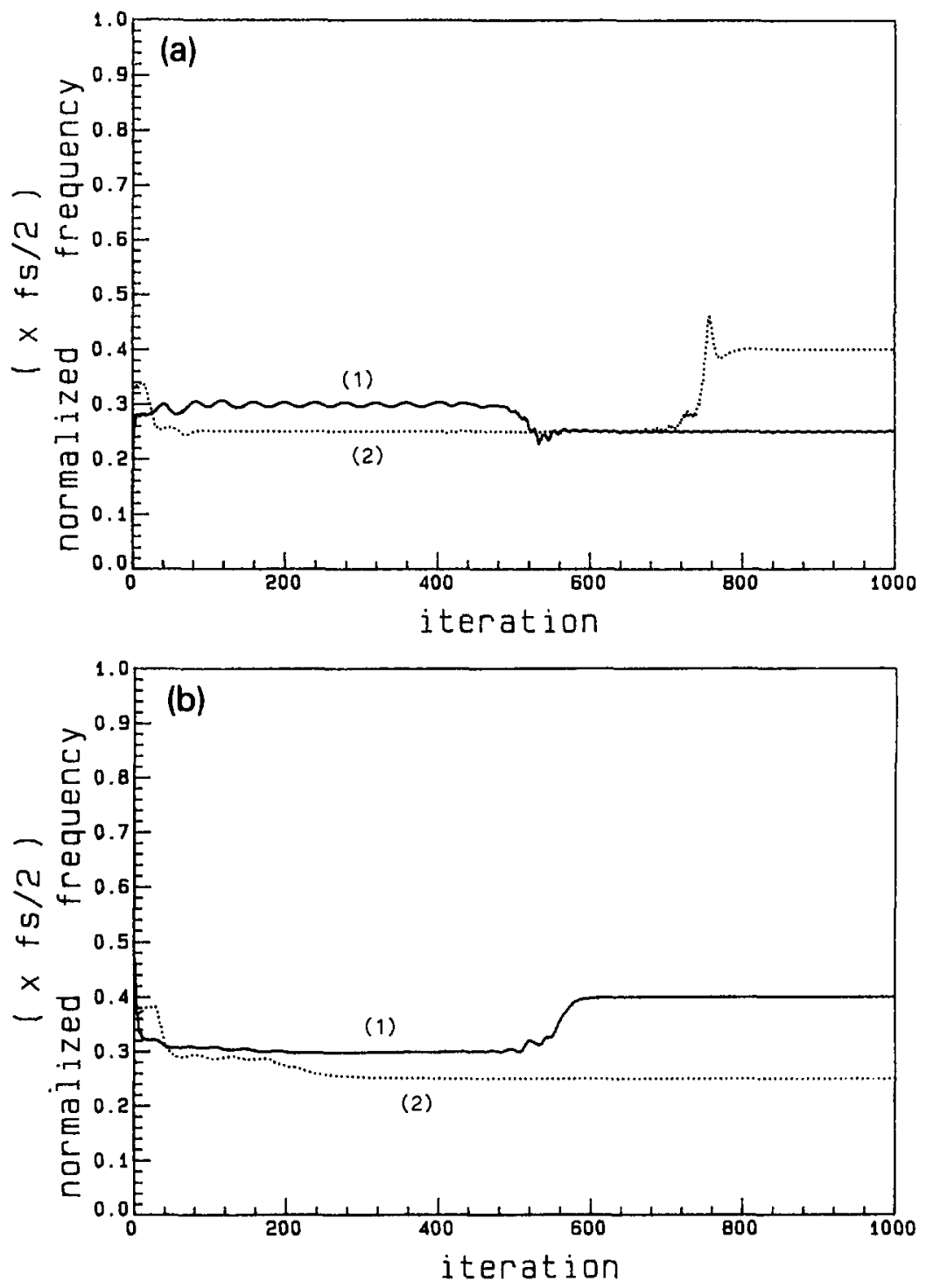

Fig. 6. (a) The learning curves of frequencies of 2 stage second-order systems for two input step changed sinusoids which are closely spaced in frequencies. [Individual adaption scheme: (1) solid line: first stage, (2) dot line: second stage.] (b) The learning curves of frequencies of 2 stage second-order systems for two input step changed sinusoids which are closely spaced in frequencies. [Simultaneous adaption scheme: (1) solid line: first stage, (2) dot line: second stage.]

(D) (Sixth-order adaptive notch filter system.) The input signal is
Refering to Fig. 8 , the first stage tracks the timevarying sinusoid switched from 0.3 to 0.25 instead

$$
y(t)= \begin{cases}\sqrt{20} \cos (0.25 \pi t)+\sqrt{20} \cos (0.3 \pi t)+\sqrt{20} \cos (0.5 \pi t)+v(t) & \text { if } t \leqslant 400 \\ \sqrt{20} \cos (0.25 \pi t)+\sqrt{20} \cos (0.4 \pi t)+\sqrt{20} \cos (0.5 \pi t)+v(t) & \text { if } t>400\end{cases}
$$




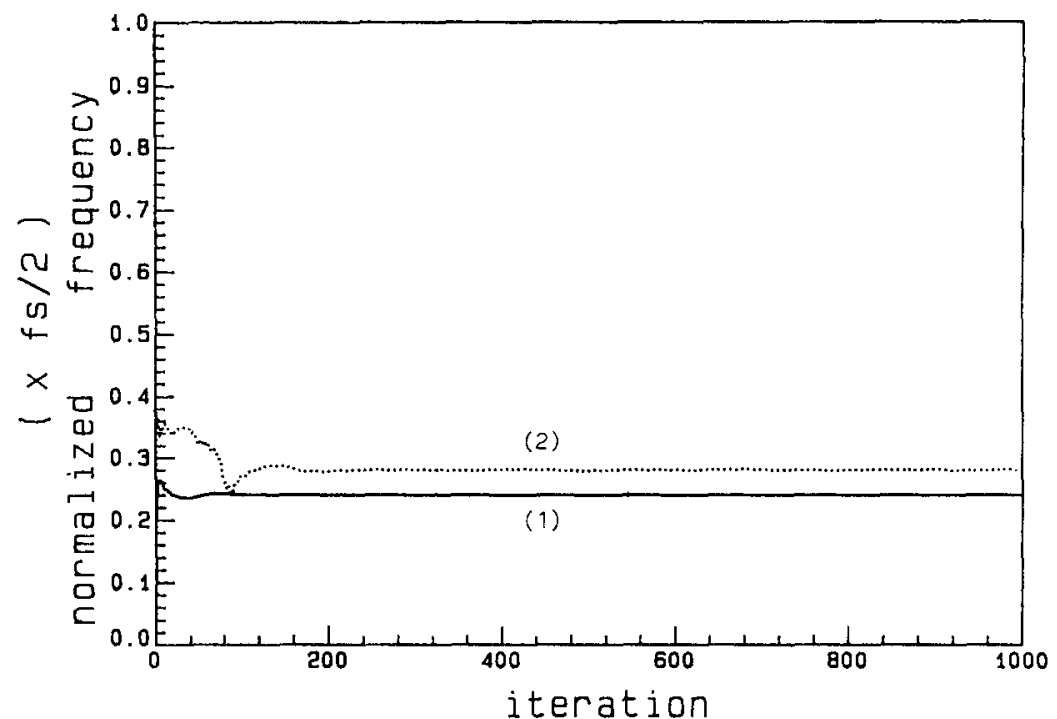

Fig. 7. The learning curves of frequencies of 2 stage second-order systems for two input sinusoids which are closely spaced in frequencies with large power difference.

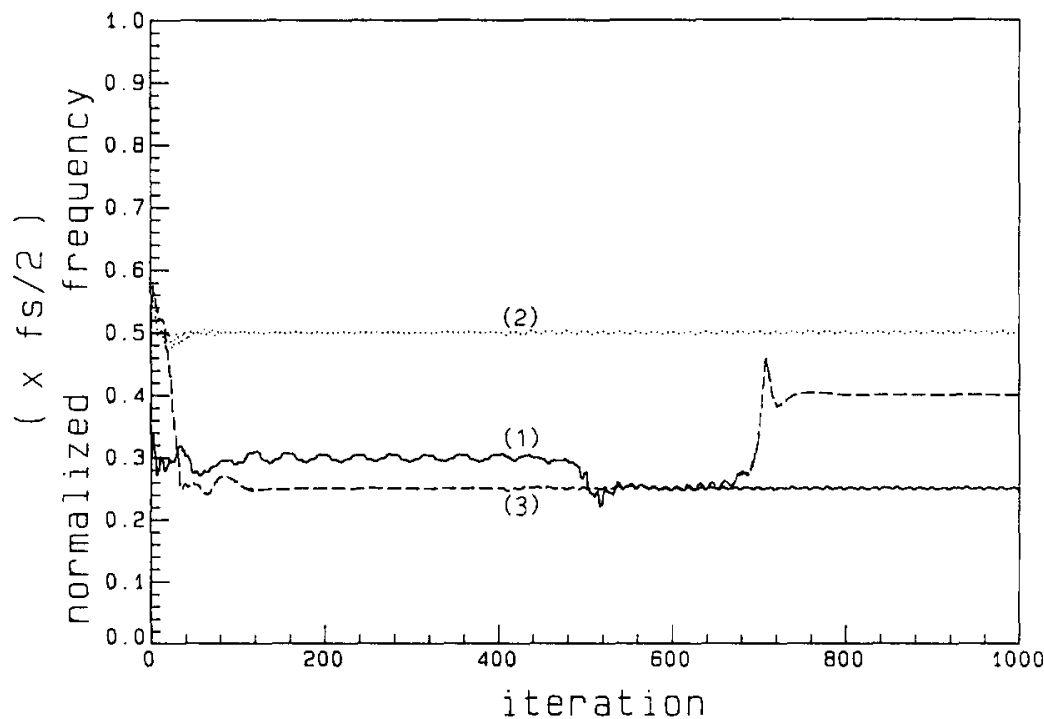

Fig. 8. The learning curves of frequencies of 3 stage second-order systems for three input step changed sinusoids which are separated in frequencies. [(1) solid line: first stage, (2) dot line: second stage, (3) dash line: third stage.]

of 0.4 . This is because the frequency at 0.3 is muchcloser to 0.25 than the frequency 0.4 . This forces the third stage to jump from 0.25 to 0.4 . A similar situation happened also in case $(B)$ as above. The second stage always stays at the distant sinusoid at 0.5 without change.

\section{Conclusion}

In this paper, we have proposed a cascade adaptive notch filter scheme for sinusoidal parameter estimation and tracking. Our scheme uses enhanced signal as reference of amplitude/phase 
estimator. This improves the accuracy of amplitude and phase estimation. Also, the RLS algorithm is simplified to adjust the amplitude and phase. Error surface analysis indicates that our scheme is unimodal and results in guaranted convergence.

\section{References}

[1] B. Friedlander and J.O. Smith, "Analysis and performance evaluations of an adaptive notch filter", IEEE Trans. Inform. Theory, Vol. IT-30, March 1984, pp. 280-295.

[2] D.R. Hush, N. Ahmed, R. David and S.D. Sterns, "An adaptive IIR structure for sinusoidal enhancement, frequency estimation and detection", IEEE Trans. Acoust. Speech Signal Process., Vol. ASSP-34, December 1986, pp. $1380-1390$.

[3] T. Kwan and K. Martin, "Adaptive detection and enhancement of multiple sinusoids using a cascade IIR filter", IEEE Trans. Circuits and Systems, Vol. CAS-36, July 1989, pp. 937-947.

[4] K. Martin, "The isolation of undistorted sinusoids in real time", IEEE Internat. Symp. on Circuit and Systems, Portland, USA, May 1989, pp. 2112-2115.
[5] N.K. M'sirdi and H.A. Tjokronegoro, "Cascade adaptive notch filters: an RML estimation algorithm", European Signal Process. Conf., Grenoble, France, September 1988, pp. 1408-1412.

[6] N.K. M'sirdi, H.A. Tjokronegoro and I.D. Landau, "An RML algorithm for retrieval of sinusoids with cascaded notch filter", 1988 Internat. Conf. Acoust. Speech Signal Process., New York, USA, April 1988, pp. 2484-2487.

[7] M. Nayeri and W.K. Jenkins, "Alternate realizations to adaptive IIR filters and properties of their performance surfaces", IEEE Trans. on Circuits and Systems, Vol. CAS36, April 1989, pp. 485-496.

[8] A. Nehorai, "A minimal parameter adaptive notch filter with constrained poles and zeros", IEEE Trans. Acoust. Speech Signal Process., Vol. ASSP-33, August 1985, pp. 983-996.

[9] T.S. Ng, "Some aspects of an adaptive digital notch filter with constrained poles and zeros", IEEE Trans. Acoust Speech Signal Process., Vol. ASSP-35, February 1987, pp. 158-161.

[10] D.V.B. Rao and S.Y. Kung, "Adaptive notch filtering for the retrieval of sinusoids in noise", IEEE Trans. Acoust. Speech Signal Process., Vol. ASSP-32, August 1984, pp. 791-802.

[11] S.D. Sterns, "Error surface of recursive adaptive filters", IEEE Trans. on Acoust. Speech Signal Process., Vol. ASSP-29, June 1981, pp. 763-766. 